\title{
Disc inflammation and Modic changes show an interaction effect on recovery after surgery for lumbar disc herniation
}

\author{
Niek Djuric ${ }^{1} \oplus$ - Xiaoyu Yang ${ }^{1} \cdot$ Raymond W. J. G. Ostelo $^{2,3} \cdot$ Sjoerd G. van Duinen ${ }^{4} \cdot$ Geert J. Lycklama à Nijeholt $^{5}$. \\ Bas F. W. van der Kallen ${ }^{4}$. Wilco C. Peul ${ }^{1,5}$. Carmen L. A. Vleggeert-Lankamp ${ }^{1}$
}

Received: 30 January 2019 / Revised: 18 July 2019 / Accepted: 7 August 2019 / Published online: 22 August 2019

(c) The Author(s) 2019

\begin{abstract}
Purpose To study the interaction between Modic changes (MC) and inflammation by macrophages in the disc, in relation to clinical symptoms before and after discectomy for lumbar disc herniation.

Methods Disc tissue was embedded in paraffin and stained with haematoxylin and CD68. Subsequently, tissue samples were categorized for degree of inflammation. Type of MC was scored on MRI at baseline. Roland Disability Questionnaire (RDQ) score and visual analogue scale for back pain and leg pain separately were considered at baseline and 1-year followup post-surgery. Main and interaction effects of MC and inflammation were tested against clinical outcome questionnaires. In addition, this analysis was repeated in bulging and extruded discs separately.

Results Disc material and MRI's of 119 patients were retrieved and analysed. Forty-eight patients demonstrated mild inflammation, 45 showed moderate inflammation, and 26 showed considerable inflammation. In total, 49 out of 119 patients demonstrated MC. Grade of disc inflammation did not associate with the presence of MC. At baseline, no main or interaction effects of MC and inflammation were found on the clinical scores. However, during follow-up after discectomy, significant interaction effects were found for RDQ score: Only in patients with MC at baseline, patients remained significantly more disabled (3.2 points $p=0.006$ ) if they showed considerable disc inflammation compared to patients with mild inflammation. The additional analysis showed similar results in extruded discs, but no significant effects in bulging discs.

Conclusions An interaction effect of MC and disc inflammation by macrophages is present. Only in patients with MC, those with considerable inflammation recover less satisfactory during follow-up after surgery.
\end{abstract}

\section{Graphic abstract}

These slides can be retrieved under Electronic Supplementary Material.
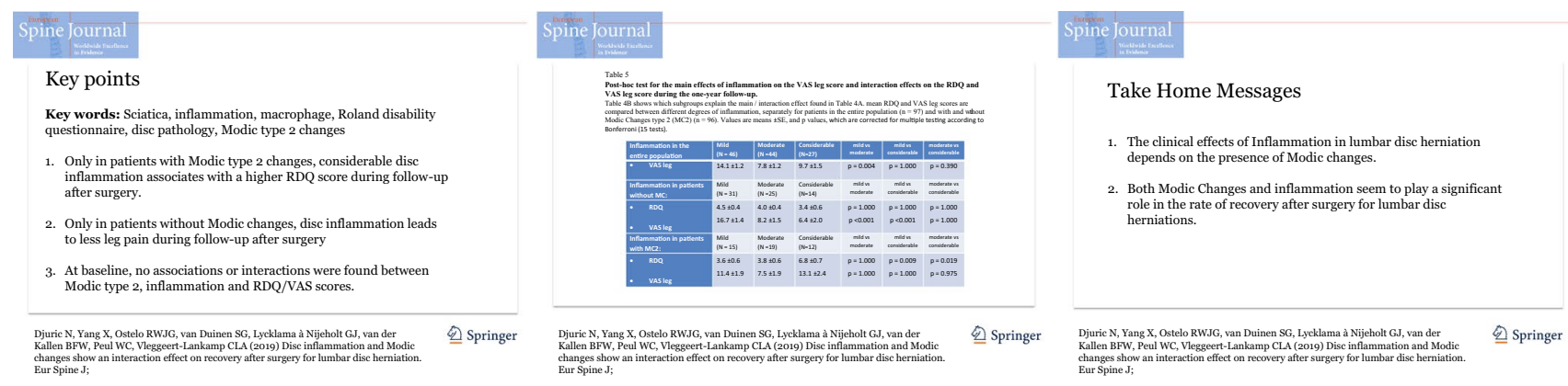

Keywords Sciatica $\cdot$ Inflammation $\cdot$ Macrophage $\cdot$ Roland disability questionnaire $\cdot$ Modic type 2 changes

Electronic supplementary material The online version of this article (https://doi.org/10.1007/s00586-019-06108-9) contains supplementary material, which is available to authorized users.

Extended author information available on the last page of the article 


\section{Introduction}

Patients with lumbar disc herniation often suffer from radicular pain symptoms, the origin of which is not fully understood. Even though a part can be explained by mechanical compression of the nerve root, inflammation also seems to play a major role. Disc inflammation may occur if the nucleus pulposus herniates into the epidural space. This may induce a foreign-body reaction accompanied by neovascularization and macrophage infiltration [1].

Macrophages may subsequently induce a resorption process by excreting matrix metalloproteases [2], inducing apoptosis and degrading collagen fibres [3]. This so-called functional inflammation response can explain spontaneous regression in herniation size [4]. On the other hand, they can also excrete pro-inflammatory cytokines such as IL-6, IL8 and TNF-alpha, which have been associated with exacerbation of the pain symptoms, the so-called painful inflammation response [5-7]. This discrepancy in inflammation response is reflected in the inconsistent findings regarding the correlation between the presence of macrophages in herniated disc material and clinical symptoms [8,9]. As of today, it remains unknown what factors are of influence on the type of inflammation response that patients experience. Nevertheless, the degree of inflammation can be influenced by specific characteristics of the disc. For example, extruded discs, which are more exposed to the systemic circulation, tend to have a higher degree of inflammation as compared to bulging discs [4]. Therefore, the type of disc herniation should not be neglected when the characteristics of disc inflammation are studied.

Modic changes (MC), also known as vertebral endplate signal changes (VESC) on MRI, are often seen in patients with lumbar disc herniation [10] and have been proposed to associate with slowing the recovery rate in patients that suffer from a herniated disc [11]. In addition, Dudli (2017) introduced the suggestion that MC represent the effect of crosstalk between bone marrow and the intervertebral disc [12]. Hence, changes in the vertebral endplate, like inflammatory dysmyelopoiesis and upregulation in neurotrophic factors, may induce the presence of different types of macrophages, which may interact with the inflammation response in the disc. By doing so, possibly, MC represent a shift in the above-mentioned inflammation reaction from 'functional' towards 'painful', thereby lowering the rate of recovery [11]. Until now, no studies have sought to enlighten this interaction. Therefore, the aim of the present study is to explore the interactions between disc inflammation and $\mathrm{MC}$ on the rate of recovery after surgery.

\section{Materials and methods}

\section{Hypothesis}

A significant interaction effect is expected between disc inflammation and MC on the clinical symptoms: Patients with MC will suffer from a high degree of inflammation, while patients without MC will benefit from inflammation. In addition, we expect this effect to be clearer in extruded discs as compared to bulging discs.

\section{Study population}

This retrospective study was performed using participants from the Sciatica Trial [13], a multicenter RCT with 283 patients who suffered from sciatica for 6-12 weeks and had a disc herniation as assessed by means of MRI. A total of 141 patients were randomized to surgery, and 125 patients actually underwent surgery (16 recovered before surgery could be performed). The other 142 patients were randomized to prolonged conservative care, of which 55 patients underwent surgery within 1 year, with a mean time to surgery of 15 weeks after randomization. Thus, in the first year after randomization, a total of 180 patients underwent surgery for sciatica. Out of the 180 patients, 120 disc samples were available for analysis. Missing samples were due to multiple reasons: not collected during surgery, got lost after surgery, not preserved properly, or got lost after preservation. All surgeries were performed between November 2002 and February 2005. The protocol, which included analysis of the disc material, was approved by the medical ethics committees at all participating hospitals.

\section{Histological analysis}

Disc material of all operated patients was collected and fixed in $4 \%$ formaldehyde solution after surgery and was subsequently stored for future analysis. For the purpose of this retrospective study, samples were embedded in blocks of paraffin and stained with CD68 to evaluate inflammation by macrophages. A detailed description of the protocol was published in our previous work [14].

The evaluation was done by two independent investigators, who were trained by a senior pathologist and were blinded to clinical information. The number of macrophages in each sample was counted semiquantitatively and categorized according to their inflammation grade. The categories consisted of mild (0-10 macrophages per $\mathrm{cm}^{2}$ ), moderate (10-100 macrophages per $\left.\mathrm{cm}^{2}\right)$, and considerable $(>100$ macrophages per $\mathrm{cm}^{2}$ ) inflammation. Subsequently, the interobserver agreement between the independent researchers 
Fig. 1 Examples of mild (0-10 macrophages per $\left.\mathrm{cm}^{2}\right)$, moderate (10-100 macrophages per $\left.\mathrm{cm}^{2}\right)$, and considerable $(>100$ macrophages per $\mathrm{cm}^{2}$ ) inflammation

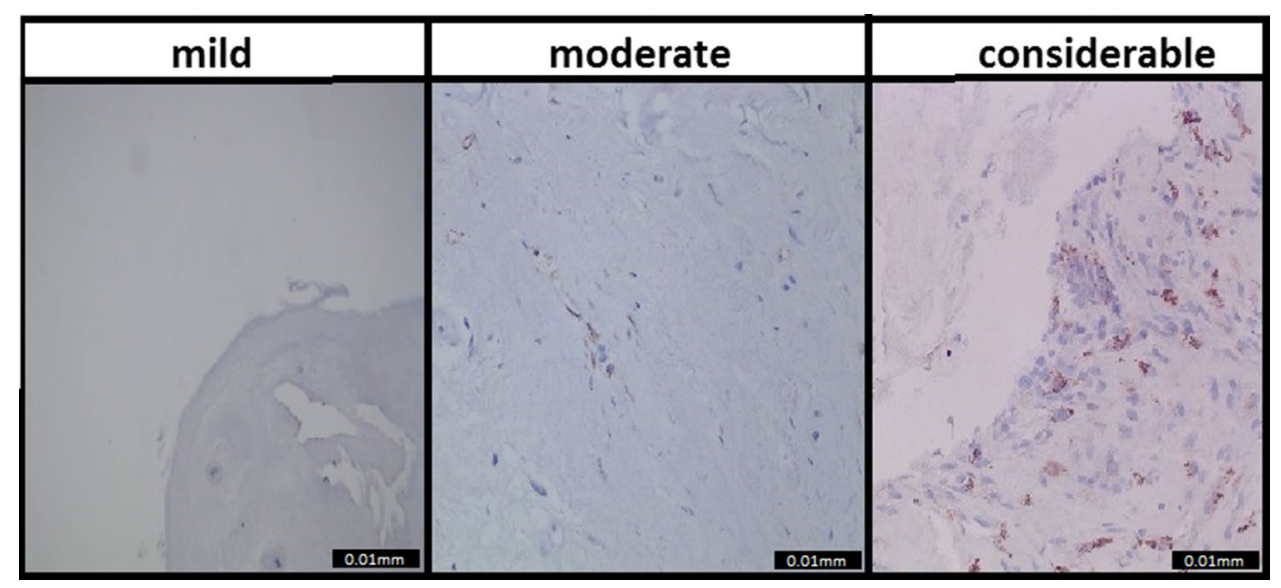

was determined. The inter-observer agreement value was predefined to be over $60 \%$. Therefore, it was scheduled to reevaluate the tissue sample if the agreement was less than $60 \%$, involving evaluation of the senior pathologist. After the consensus reading, a consensus score was calculated.

\section{MRI analysis for MC}

MRI scans were performed at baseline by a 1.5 -Tesla scanner, and both sagittal T1- and T2-weighted images of the lumbar spine were evaluated. According to the criteria of Modic et al. [15, 16], Modic changes were scored as Type 1, Type 2 , or Type 3 . Type of disc herniation as quantified by MRI was categorized as follows: bulging, extrusion, sequestration or not applicable. The definition of protrusion as defined by the protocol is more commonly known as 'bulging' in daily clinical practice. Therefore, this term is used in this article. MRIs were evaluated by two neuroradiologists and one neurosurgeon. All three were blinded to histological data and clinical information. The readers were not involved in the selection or treatment of the patients included. Interobserver agreement analysis was published earlier by el Barzouhi et al. [17]. Because in earlier findings no MC were observed at level L1-L2, only images from L2-L3 through L5-S1 were evaluated. For the statistical analyses, the majority opinion of the three independent researchers (answer by at least two of the three MRI assessors) was used.

\section{Clinical outcome}

The clinical outcome parameters from the Sciatica trial that we used to associate the histological data with were the Roland Disability Questionnaire (RDQ): the scores ranging from 0 to 23, with higher scores indicating worse functional status [18], and the 100-mm visual analogue scale (VAS) for leg and back pain: with 0 representing no pain and 100 the worst pain ever experienced [19]. These outcome measures were considered at baseline, and at 2, 4, 8, 12, 26, 38,
52 weeks post-surgery. For the RDQ score, a difference of 3 points was regarded as the minimal clinically important change [20]. For the VAS score, this threshold was set at 19 points [21].

\section{Statistical analysis}

First, the presence of MC was tested against the dichotomized histological findings and against the type of disc herniation using Chi-square tests for categorical data. The association between disc type and inflammation was previously reported [4]. For the clinical analysis at both baseline and follow-up, patients were first grouped based on the type of herniation, Subsequently, the main effects of inflammation and MC, and their interaction effect were tested against the clinical baseline scores (RDQ, VAS leg pain and VAS back pain). For this analysis, a two-way ANOVA was used.

In order to analyse the predictive value of inflammation and $\mathrm{MC}$ at baseline, these were tested for associations with clinical outcomes (VAS back, VAS leg and RDQ) over the course of 1-year follow-up using a mixed model analysis. The clinical outcomes were measured at 2, 4, 8 12, 26, 36 and 52 weeks post-surgery. For both baseline and followup analyses, Bonferroni correction for multiple testing was used. If additional post hoc tests were indicated, those were corrected for the number of post hoc tests. $P$ values of $<0.05$ were regarded as significant.

\section{Results}

\section{The histological data analysis}

CD68 staining to identify macrophages resulted in the following distribution: 48 (40\%) patients were scored as mild inflammation, 45 (37.5\%) patients as moderate inflammation, and 27 (22.5\%) patients as considerable inflammation. 
Table 1 Baseline characteristics of the three histologically defined inflammation groups for patients with and without Modic type 2 changes

\begin{tabular}{|c|c|c|c|c|c|c|}
\hline & $\begin{array}{l}\text { No/mild } \\
\text { (without MC) } \\
(N=31)\end{array}$ & $\begin{array}{l}\text { No/mild (with } \\
\mathrm{MC})(N=16)\end{array}$ & $\begin{array}{l}\text { Moderate } \\
\text { (without MC) } \\
(N=25)\end{array}$ & $\begin{array}{l}\text { Moderate } \\
\text { (with MC) }(N=19)\end{array}$ & $\begin{array}{l}\text { Strong } \\
\text { (without MC) }(N=14)\end{array}$ & $\begin{array}{l}\text { Strong } \\
(\text { With MC) }(N=12)\end{array}$ \\
\hline Age & $39.0 \pm 8.9$ & $43.1 \pm 10.7$ & $39.8 \pm 9.4$ & $45.1 \pm 11.1$ & $40.7 \pm 6.5$ & $47.8 \pm 4.4$ \\
\hline Male $(\%)$ & $83.9 \%$ & $62.5 \%$ & $52 \%$ & $73.7 \%$ & $71.4 \%$ & $100 \%$ \\
\hline Body mass index & $26.0 \pm 4.3$ & $26.3 \pm 3.7$ & $25.2 \pm 3.8$ & $26.0 \pm 2.7$ & $24.2 \pm 2.3$ & $27.6 \pm 3.0$ \\
\hline $\begin{array}{l}\text { Duration of sciatica in } \\
\text { weeks }\end{array}$ & $9.4 \pm 1.8$ & $10.0 \pm 2.2$ & $9.5 \pm 2.5$ & $9.8 \pm 2.3$ & $8.6 \pm 2.3$ & $8.5 \pm 1.9$ \\
\hline Roland Disability score & $16.5 \pm 4.8$ & $16.2 \pm 3.6$ & $17.0 \pm 4.2$ & $17.3 \pm 3.4$ & $16.6 \pm 4.3$ & $16.5 \pm 5.1$ \\
\hline VAS leg pain & $64.6 \pm 22.5$ & $63.0 \pm 20.6$ & $69.9 \pm 16.7$ & $61.1 \pm 25.2$ & $76.4 \pm 10.4$ & $67.3 \pm 22.1$ \\
\hline VAS back pain & $27.8 \pm 31.7$ & $46.6 \pm 32.8$ & $40.5 \pm 27.4$ & $28.3 \pm 27.8$ & $32.8 \pm 28.0$ & $21.3 \pm 30.8$ \\
\hline
\end{tabular}

From one of the patients no data on Modic changes at baseline could be retrieved, Therefore the group of patients with "considerable inflammation' is decreased to 26 patients. Values are presented as mean $\pm \mathrm{SD}$ (except for the male/female ratio)

The consensus score was excellent (0.96) [6]. Examples of the CD68 samples and their categories are shown in Fig. 1.

\section{MRI analysis for MC and disc type}

Of the 120 patients included, one patient's MRI was lost, two MRI's showed MC type 1 changes (MC1) and 47 showed MC type 2 (MC2), of which 42 at the level of the disc herniation, and 70 showed no MC (inter-observer agreement 69-97\%). Because only two patients demonstrated MC1, this subgroup was not suitable for statistical analysis. Hence, they were excluded from the analyses. Regarding disc type, 40 discs were characterized as bulging disc and 73 as extruded disc, no sequestrations were seen $(\mathrm{kappa}=0.62)$.

\section{Clinical outcome}

Baseline characteristics age, gender, BMI and duration of sciatica symptoms prior to surgery in the three inflammation groups were comparable (Table 1). After surgery, VAS leg and back pain and RDQ scores decreased significantly in all patients.

\section{Association between inflammation grade, and clinical outcome at baseline}

At baseline, no significant association was found between the grade of inflammation and the presence of MC2 $(p=0.525)$, nor between MC2 and disc type $(p=0.239)$ (Table 2). As described earlier, a positive association was found between bulging discs and the degree of inflammation [4].

No significant main or interaction effects were found between MC2 and inflammation by macrophages with either RDQ, nor with VAS leg pain nor with VAS back pain scores at baseline (Table 3 ). These results did not change
Table 2 Associations between MC and inflammation at baseline

\begin{tabular}{lccc}
\hline$X^{2}$ test & No MC & MC2 & $P$ value \\
\hline Inflammation & & & 0.525 \\
Mild $(n=48)$ & 25 & 16 & \\
Moderate $(n=45)$ & 14 & 12 & \\
Considerable $(n=26)$ & & & 0324 \\
Type of herniation & 1 & 2 & \\
Not applicable (3) & 27 & 13 & \\
Bulging (40) & 41 & 32 & \\
Extrusion $(73)$ & 0 & 0 & \\
Sequestration & &
\end{tabular}

Values are $n . P$ values of the Chi-square test are given

when bulging and extruded discs were considered separately (Table S1).

\section{Association between inflammation grade and clinical outcome at follow-up}

Clinical outcome during follow-up was measured at the time points $2,4,8,12,26,38$ and 52 weeks after surgery. However, the timing of surgery was not equal for all 117 patients. In the sciatica RCT, about $40 \%$ of patients randomized to conservative treatment, crossed over to surgical treatment because of unbearable symptoms. These patients were clinically evaluated at the same time points after randomization. However, surgical intervention was performed with a mean of delay 15 weeks, ranging from 15 days to 18 months after randomization. Mean waiting time prior to randomization was 9.4 weeks. In our sample group, 34 of 120 patients were originally randomized to conservative care and crossed over. The measurements at time points 2, 4, 8, 12, 26, 38 and 52 after randomization were likely influenced by surgical intervention effects. Therefore, patients with a deviation of more 
Table 3 Baseline main and interaction effects of MC2 and inflammation on clinical outcomes

\begin{tabular}{llll}
\hline $\begin{array}{l}\text { Mixed model test (patients } \\
\text { with Modic changes): }\end{array}$ & RDQ core F value $(p$ value $)$ & $\begin{array}{l}\text { VAS leg score } F \text { value } \\
(p \text { value })\end{array}$ & $\begin{array}{l}\text { VAS back score } \\
F \text { value }(p \\
\text { value })\end{array}$ \\
\hline MC2 effect & 0.01 & 2.61 & 0.78 \\
Inflammation effect & $(p=1.000)$ & $(p=1.000)$ & $(p=1.000)$ \\
$\begin{array}{l}\text { Interaction effect MC2* } \\
\text { inflammation }\end{array}$ & $0.34(p=1.000)$ & $1.25(p=1.000)$ & $0.40(p=1.000)$ \\
\hline
\end{tabular}

Two-way ANOVA was used, values are $F$ values with $p$ values, $n$ total $=119 . P$ values are corrected for multiple testing according to Bonferroni (9 tests)
Table 4 Follow-up main and interaction effects of MC2 and inflammation on clinical outcome

\begin{tabular}{llll}
\hline $\begin{array}{l}\text { Mixed model } \\
\text { test (patients } \\
\text { with Modic } \\
\text { changes): }\end{array}$ & $\begin{array}{l}\text { RDQ score } F \\
\text { value }(p \text { value })\end{array}$ & $\begin{array}{l}\text { VAS leg score } \\
F \text { value }(p \\
\text { value })\end{array}$ & $\begin{array}{l}\text { VAS back score } \\
F \text { value }(p \\
\text { value })\end{array}$ \\
\hline MC2 effect & 2.47 & 0.02 & 1.52 \\
Inflammation & $(p=0.963)$ & $(p=1.000)$ & $(p=1.000)$ \\
$\quad$ effect & $2.25(p=1.000)$ & & \\
$\begin{array}{c}\text { Interaction } \\
\text { effect MC2* }\end{array}$ & $7.10(p=0.009)$ & $6.97(p=0.009)$ & $2.32(p=0.891)$ \\
inflammation & & $4.69(p=0.090)$ & $0.75(p=1.000)$ \\
\hline
\end{tabular}

Mixed model analysis was used, values are $F$ values with $p$ values, $n$ total $=96$. $P$ values are corrected for multiple testing according to Bonferroni ( 9 tests)

than 3 SD's 'delay to surgery time' were considered outliers and excluded from the analysis. Twenty-one of the 34 patients were therefore excluded; 13 patients that remained had a mean waiting time for surgery of 25 days with a range of 2-51. The clinical data of the time points before the surgical intervention were ignored.
Over the course of 1-year follow-up after surgery, no significant main effects were found of inflammation or MC2 on the RDQ score. However, a significant interaction effect was present $(p=0.009)$, indicating that the association between inflammation and the RDQ score depended on the presence or absence of MC2. Post hoc tests revealed that in the patient subgroup without MC, no significant associations between inflammation and RDQ score were found. On the contrary, in patients with $\mathrm{MC}$, those with a considerable inflammation had significantly and clinically relevant higher RDQ scores (mean $=6.8)$ compared to those with moderate- $($ mean $=3.8$, $p=0.019)$ or mild inflammation $($ mean $=3.6, p=0.009)$ (Tables 4, 5, Fig. 2a, b).

Furthermore, a significant association was found between inflammation and the VAS leg pain score during follow-up $(p=0.009)$ (Tables 4, 5). However, when assessing the interaction effect between MC2 and inflammation, this association between inflammation on the VAS leg score was only seen the patient subgroup without MC, in which the patients with mild inflammation experienced significantly higher VAS leg score $($ mean $=16.7)$ compared to both the moderate $($ mean $=8.2, p<0.001)$ and considerable inflammation

Table 5 Post-hoc test for the main effects of inflammation on the VAS leg score and interaction effects on the RDQ and VAS leg score during the 1-year follow-up

\begin{tabular}{|c|c|c|c|c|c|c|}
\hline $\begin{array}{l}\text { Inflammation in the } \\
\text { entire population }\end{array}$ & Mild $(N=46)$ & Moderate $(N=44)$ & $\begin{array}{l}\text { Considerable } \\
(N=27)\end{array}$ & $\begin{array}{l}\text { Mild versus } \\
\text { moderate }\end{array}$ & $\begin{array}{l}\text { Mild versus } \\
\text { considerable }\end{array}$ & $\begin{array}{l}\text { Moderate versus } \\
\text { considerable }\end{array}$ \\
\hline VAS leg & $14.1(11.7 ; 16.4)$ & $7.8(5.5 ; 10.2)$ & $9.7(6.7 ; 12.7)$ & $p=0.004$ & $p=1.000$ & $p=0.390$ \\
\hline $\begin{array}{l}\text { Inflammation in } \\
\text { patients without } \\
\text { MC }\end{array}$ & $\begin{array}{l}\text { Mild } \\
(N=31)\end{array}$ & $\begin{array}{l}\text { Moderate } \\
(N=25)\end{array}$ & $\begin{array}{l}\text { Considerable } \\
(N=14)\end{array}$ & $\begin{array}{l}\text { Mild versus } \\
\text { moderate }\end{array}$ & $\begin{array}{l}\text { Mild versus } \\
\text { considerable }\end{array}$ & $\begin{array}{l}\text { Moderate versus } \\
\text { considerable }\end{array}$ \\
\hline RDQ & $4.5(3.7 ; 5.4)$ & $4.0(3.2 ; 4.9)$ & $3.4(2.3 ; 4.6)$ & $p=1.000$ & $p=1.000$ & $p=1.000$ \\
\hline VAS leg & $16.7(13.9 ; 19.5)$ & $8.2(5.1 ; 11.1)$ & $6.4(2.6 ; 10.3)$ & $p<0.001$ & $p<0.001$ & $p=1.000$ \\
\hline $\begin{array}{l}\text { Inflammation in } \\
\text { patients with MC2 }\end{array}$ & Mild $(N=15)$ & Moderate $(N=19)$ & $\begin{array}{l}\text { Considerable } \\
(N=12)\end{array}$ & $\begin{array}{l}\text { Mild versus } \\
\text { moderate }\end{array}$ & $\begin{array}{l}\text { Mild versus } \\
\text { considerable }\end{array}$ & $\begin{array}{l}\text { Moderate versus } \\
\text { considerable }\end{array}$ \\
\hline RDQ & $3.6(2.4 ; 4.8)$ & $3.8(2.7 ; 5.0)$ & $6.8(5.4 ; 8.1)$ & $p=1.000$ & $p=0.009$ & $p=0.019$ \\
\hline VAS leg & $11.4(7.6 ; 15.2)$ & $7.5(3.7 ; 11.2)$ & $13.1(8.4 ; 17.7)$ & $p=1.000$ & $p=1.000$ & $p=0.975$ \\
\hline
\end{tabular}

Which subgroups explain the main/interaction effect found in Table 4. mean RDQ and VAS leg scores are compared between different degrees of inflammation, separately for patients in the entire population $(n=97)$ and with and without MC2 $(n=96)$. Values are means $($ Confidence Intervals), and $p$ values, which are corrected for multiple testing according to Bonferroni (15 tests) 
Fig. 2 a The effect of inflammation in patients without Modic changes on the RDQ score during the 1-year follow-up analysis with multiple time points. Error bars are SE'. No significant differences were seen. b The effect of inflammation in patients with Modic type 2 changes on the RDQ score during the 1-year follow-up analysis with multiple time points. Error bars are SE's. A significant difference was seen between mild and considerable, and between moderate and considerable inflammation

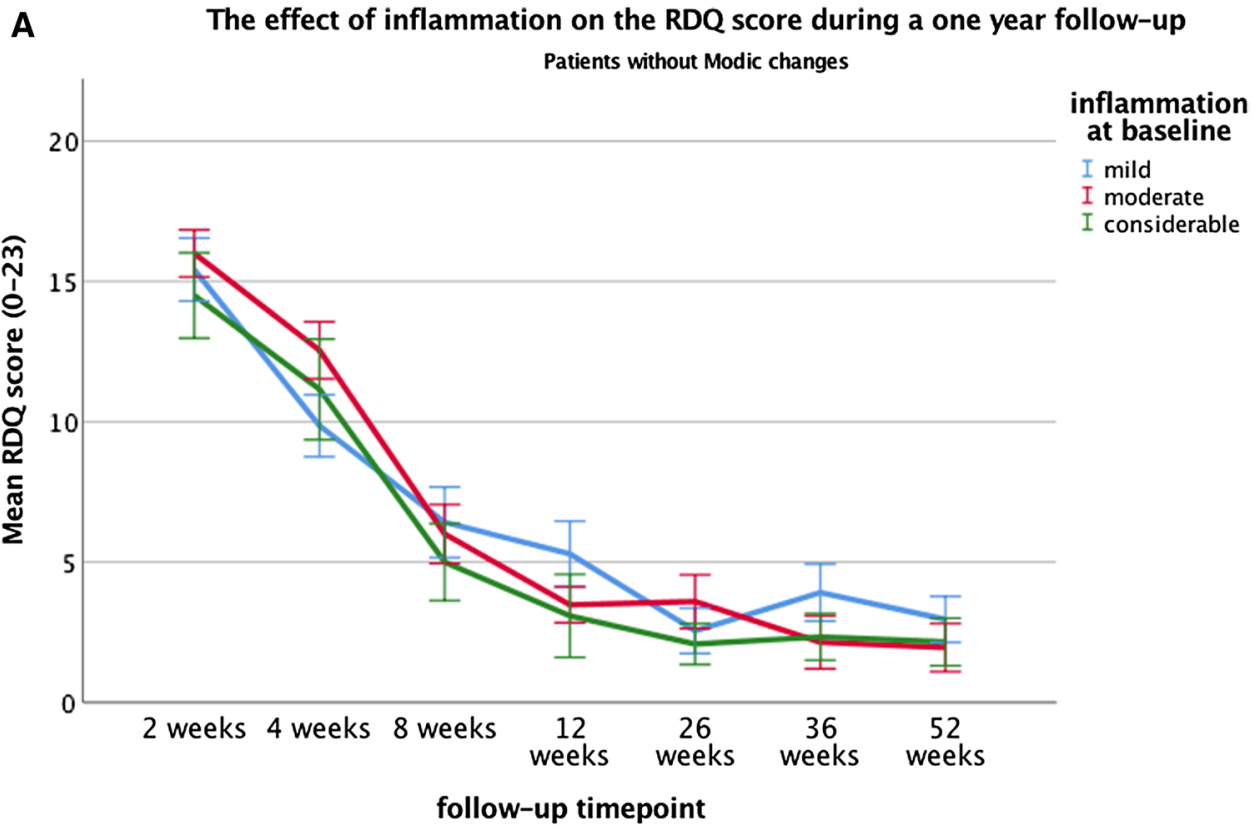

B

The effect of inflammation on the RDQ score during a one year follow-up

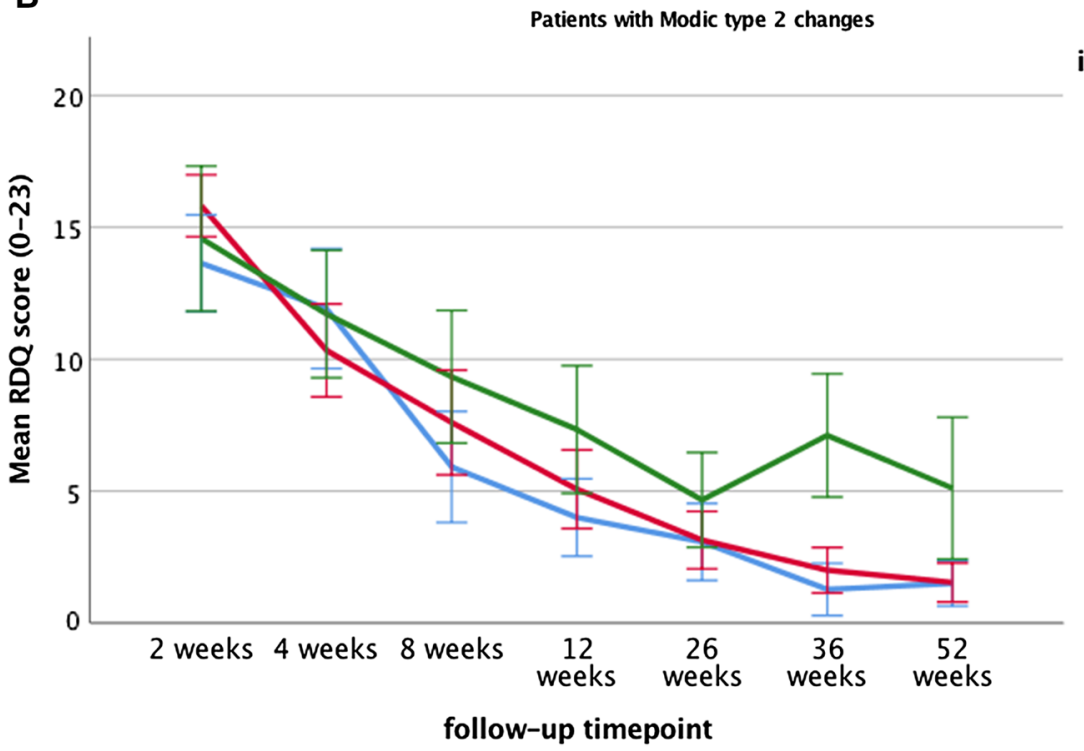

inflammation at baseline

I mild

I moderate
I considerable (mean $=6.4, p<0.001)$. In contrast, no significant associations between inflammation and VAS leg scores were found in the subgroup of patients with MC2 (Table 5, Fig. 3a, b). Even though these associations were significant, they did not exceed the clinically relevant threshold of 19 points [21]. Moreover, no significant main effect of MC2 was found on VAS leg score.

At last, no significant associations were found between inflammation and VAS back scores or between MC2 and VAS back scores. Also, no interaction effects were found, indicating that the no associations between inflammation and VAS back score were seen in both patients with and without MC2 (Table 4).
When bulging discs and extruded discs were considered separately, extruded discs illustrated similar significant interaction effects between MC2 and inflammation on the RDQ $(p=0,003)$ and VAS leg score $(P=0,05)$ (Table S1), post hoc tests also revealed similar mean differences (Table S2). In contrast, no significant effects or interactions were seen in bulging discs. 
Fig. 3 a The effect of inflammation in patients without Modic changes on the VAS leg score during the 1-year follow-up analysis with multiple time points. Error bars are SE's. A significant difference was seen between mild and moderate, and between mild and considerable inflammation. b The effect of inflammation in patients with Modic type 2 changes on the VAS leg score during the 1-year follow-up analysis with multiple time points. Error bars are SE's. No significant differences were found
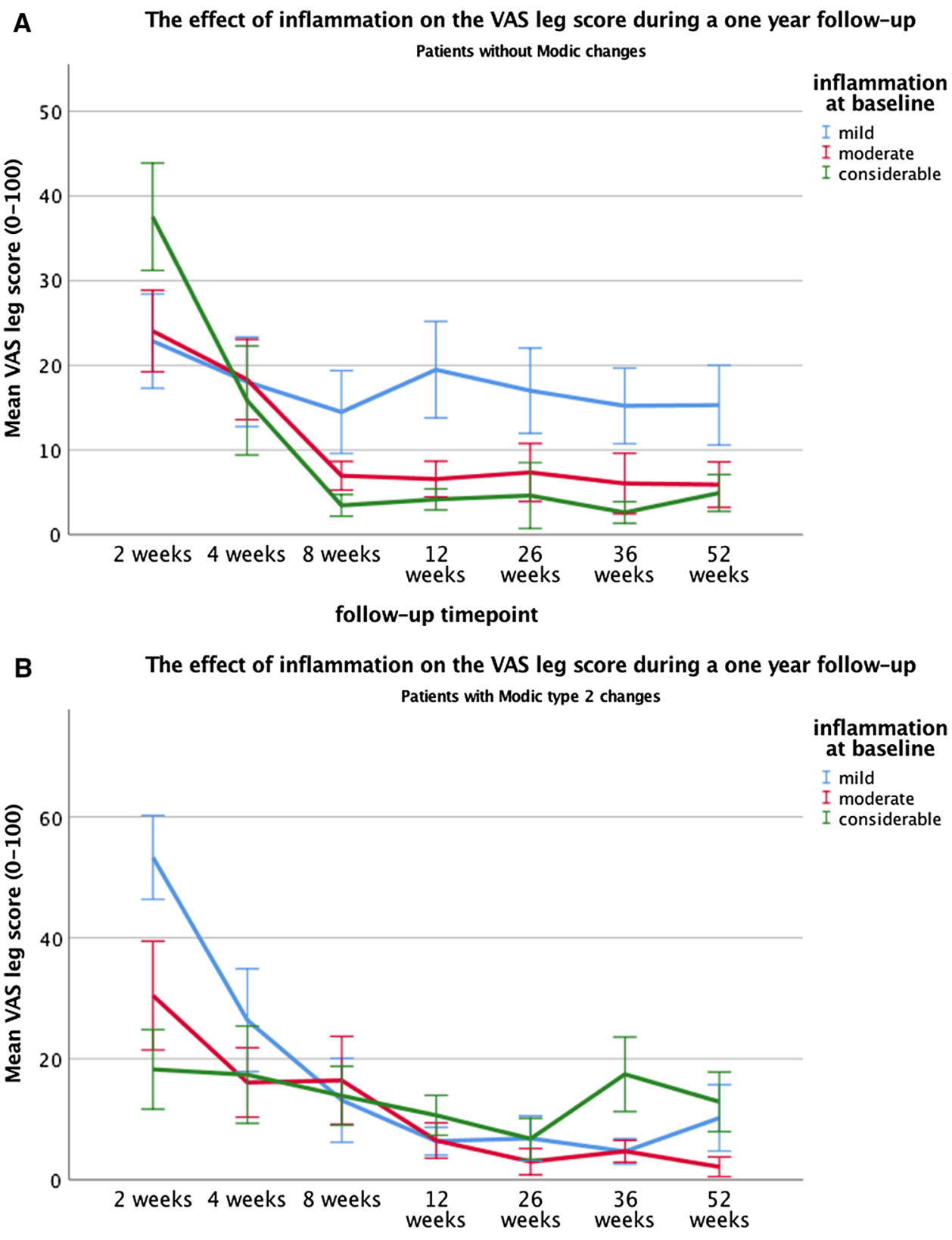

follow-up timepoint

\section{Discussion}

The present study demonstrates that the association between inflammation, as identified through macrophage infiltration, and clinical outcome after lumbar discectomy depends on the presence or absence of MC2. Patients with MC2 and considerable inflammation recovered less satisfactory after surgery in terms of disability compared to MC2 patients with mild inflammation. These results were not seen in patients without MC. In addition, in patients without MC, leg pain decreased slightly more in those with considerable inflammation as compared to those with mild inflammation.
This association was not seen in patients with MC2. Interestingly, when these bulging and extruded discs were considered separately, the above-mentioned results were only seen in extruded discs; in bulging discs, no significant effects were found. These results were in line with our hypothesis.

Here, inflammation was characterized by the presence of macrophages identified in disc tissue. As mentioned in the introduction, the type of macrophage responses can be characterized as either a 'painful inflammation response' or a 'functional inflammation response'. This contradictory effect is likely due to the difference in macrophage differentiation; M1 macrophages will act pro-inflammatory, 
while M2 macrophages are involved in resorption [5]. Based on our results, we expect a high percentage of M2 macrophages in patients without MC. In contrast, a high percentage of M1 macrophages can be expected in patients with MC2. The alternative differentiation of macrophages could possibly be explained due to the exposure to outside factors. In patients without MC, this will be limited to macrophage infiltration from the epidural space, but in patients with $\mathrm{MC}$, the disc will likely also be exposed to inflammatory factors excreted from the endplate [12]. Markers such as CSF1, CCL2, IL-1 $\beta$ and IL6 could subsequently lead to the macrophage differentiation towards M1 $[5,12]$. In addition, since more macrophages are present in extruded discs, any interaction with the endplate excretion products will be more noticeable, which is illustrated by the fact that the studied effects were most prominent in discs with considerable inflammation. This may also explain the absence of significant effects in bulging discs, where considerable inflammation was very scarce and could thus not be analysed. Taken together, this insinuates that a threshold value of inflammation has to be reached before the interaction with MC2 becomes clinically relevant, which is unlikely occur in bulging discs, since they are less exposed to the epidural space. Nevertheless, it should be noted that the number of bulging discs in this study was low, and hence, the absence of effects in bulging discs could also be due to a limited number of samples in this subgroup.

An additional factor that may lead to macrophage differentiation towards M1 might be a bacterial infection of Propionibacterium Acnes, which has been associated with MC in disc herniation patients [22]. Inferences have been made that this underlying infection may cause the difference in inflammation profile [23], and thus for macrophage differentiation towards M1. Moreover, when Propionibacterium acnes is phagocytized by a macrophage, the bacteria can disrupt its lysosomal activity and remain latent for an extended period of time [24]. P. Acnes has also been known for creating a biofilm, which does not only increase the difficulty of detecting the bacteria, but also protects them from both host immune defenses and antibiotics treatments [25]. This increased tolerance to antibiotics could also explain how $P$. acnes can survive perioperative antibiotic prophylaxis. Taken together, an underlying infection of $P$. acnes could explain both the chronic inflammation process seen on MRI as MC, and the pathological effect that macrophage infiltration can have on recovery after surgery [26]. Unfortunately, studying the different types of macrophages or bacterial presence was beyond the scope of this study.

This study has some limitations: Samples were preserved in formaldehyde for many years, which may have reduced the quality. Nevertheless, the CD68 staining showed clearly identifiable macrophages, indicating that it was not a significant issue. Furthermore, only the number of macrophages and not $\mathrm{T}$ or $\mathrm{B}$ cells were used to study inflammation. This can be justified by previous findings, which identified macrophages as the main type of inflammatory cells in discus samples [8,27], which makes them a reliable indicator of inflammation. At last, this study did not perform MRI scans with fat-saturated T2 sequences, which are the most sensitive for detecting bone marrow oedema and thus MC1 [28]. Hence, the found percentage of MC1 may be underestimated.

More importantly, in future studies, the dynamics of M1 and M2 macrophages in relation to bacterial infection of the disc and MC should be explored. Better understanding these may lead to a more accurate diagnosis, prognosis and treatment.

Acknowledgements The authors thank Stefan Hoyng for his training of the researchers in cell-counting, and Ingrid Hegeman and Annemarie Sinke for the preparation and staining of the samples.

\section{Compliance with ethical standards}

Conflict of interest None of the authors has any conflict of interest. No funding was received for the conductance of this study.

Open Access This article is distributed under the terms of the Creative Commons Attribution 4.0 International License (http://creativeco mmons.org/licenses/by/4.0/), which permits unrestricted use, distribution, and reproduction in any medium, provided you give appropriate credit to the original author(s) and the source, provide a link to the Creative Commons license, and indicate if changes were made.

\section{References}

1. Lotz JC, Fields AJ, Liebenberg EC (2013) The role of the vertebral end plate in low back pain. Global Spine J 3(3):153-163

2. Doita M, Kanatani T, Ozaki T et al (2001) Influence of macrophage infiltration of herniated disc tissue on the production of matrix metalloproteinases leading to disc resorption. Spine (Phila Pa 1976) 26(14):1522-1527

3. Kobayashi S, Meir A, Takeno K, Miyazaki T, Uchida K, Baba $\mathrm{H}$ (2009) Apoptosis in herniated disc resorption. Role of macrophages. In: 55th annual meeting of the orthopaedic research society

4. Djuric NXY, el Barzouhi A, Ostelo R, van Duinen SG, Lycklama à Nijeholt GJ, van der Kallen BFW, Peul WC, Vleggeert-Lankamp CLA (2019) Lumbar disc extrusions reduce faster than bulging disc due to an active role of macrophages in sciatica. Unpublished results

5. Duque AG, Descoteaux A (2014) Macrophage cytokines: involvement in immunity and infectious diseases. Front Immunol 5:491

6. Pedersen LM, Schistad E, Jacobsen LM et al (2015) Serum levels of the pro-inflammatory interleukins 6 (IL-6) and -8 (IL-8) in patients with lumbar radicular pain due to disc herniation: a 12-month prospective study. Brain Behav Immun 46:132-136

7. Zu B, Pan H, Zhang XJ et al (2016) Serum levels of the inflammatory cytokines in patients with lumbar radicular pain due to disc herniation. Asian Spine J 10(5):843-849 
8. Lohr M, Lebenheim L, Berg F et al (2014) Gadolinium enhancement in newly diagnosed patients with lumbar disc herniations are associated with inflammatory peridiscal tissue reactions-evidence of fragment degradation? Clin Neurol Neurosurg 119:28-34

9. Rothoerl RD, Woertgen C, Brawanski A (2002) Pain resolution after lumbar disc surgery is influenced by macrophage tissue infiltration. A prospective consecutive study on 177 patients. J Clin Neurosci 9(6):633-636

10. el Barzouhi A, Vleggeert-Lankamp CL, van der Kallen BF et al (2014) Back pain's association with vertebral end-plate signal changes in sciatica. Spine J 14(2):225-233

11. Ding L, Teng X, Fan S et al (2015) The Association between modic changes of lumbar endplates and spontaneous absorption of herniated intervertebral discs. Cell Biochem Biophys 71(3):1357-1363

12. Dudli S, Sing DC, Hu SS et al (2017) ISSLS PRIZE IN BASIC SCIENCE 2017: intervertebral disc/bone marrow cross-talk with Modic changes. Eur Spine J 26(5):1362-1373

13. Peul WC, van Houwelingen HC, van den Hout WB et al (2007) Surgery versus prolonged conservative treatment for sciatica. N Engl J Med 356(22):2245-2256

14. Djuric N, Yang X, Barzouhi AE et al (2019) Gadolinium enhancement is not associated with disc inflammation in patients with sciatica. Spine (Phila Pa 1976) 44:E742-E748

15. Modic MT, Masaryk TJ, Ross JS et al (1988) Imaging of degenerative disk disease. Radiology 168(1):177-186

16. Modic MT, Steinberg PM, Ross JS et al (1988) Degenerative disk disease: assessment of changes in vertebral body marrow with MR imaging. Radiology 166(1 Pt 1):193-199

17. El Barzouhi A, Vleggeert-Lankamp CL, Lycklama ANGJ et al (2013) Magnetic resonance imaging interpretation in patients with sciatica who are potential candidates for lumbar disc surgery. PLoS ONE 8(7):e68411

18. Patrick DL, Deyo RA, Atlas SJ et al (1995) Assessing healthrelated quality of life in patients with sciatica. Spine (Phila Pa 1976) 20(17):1899-1908 (discussion 1909)

19. Collins SL, Moore RA, McQuay HJ (1997) The visual analogue pain intensity scale: what is moderate pain in millimetres? Pain 72(1-2):95-97
20. Bombardier C, Hayden J, Beaton DE (2001) Minimal clinically important difference. Low back pain: outcome measures. J Rheumatol 28(2):431-438

21. Hagg O, Fritzell P, Nordwall A et al (2003) The clinical importance of changes in outcome scores after treatment for chronic low back pain. Eur Spine J 12(1):12-20

22. Aghazadeh J, Salehpour F, Ziaeii E et al (2017) Modic changes in the adjacent vertebrae due to disc material infection with Propionibacterium acnes in patients with lumbar disc herniation. Eur Spine J 26(12):3129-3134

23. Dudli S, Miller S, Demir-Deviren S et al (2017) Inflammatory response of disc cells against Propionibacterium acnes depends on the presence of lumbar Modic changes. Eur Spine J 27:1013-1020

24. Fischer N, Mak TN, Shinohara DB et al (2013) Deciphering the intracellular fate of Propionibacterium acnes in macrophages. Biomed Res Int 2013:603046

25. Ohrt-Nissen S, Fritz BG, Walbom J et al (2018) Bacterial biofilms: a possible mechanism for chronic infection in patients with lumbar disc herniation-a prospective proof-of-concept study using fluorescence in situ hybridization. Apmis 126(5):440-447

26. Shinohara DB, Vaghasia AM, Yu SH et al (2013) A mouse model of chronic prostatic inflammation using a human prostate cancer-derived isolate of Propionibacterium acnes. Prostate 73(9):1007-1015

27. Habtemariam A, Gronblad M, Virri J et al (1988) A comparative immunohistochemical study of inflammatory cells in acutestage and chronic-stage disc herniations. Spine (Phila Pa 1976) 23(20):2159-2165 (discussion 2166)

28. Sirvanci M, Kara B, Duran C et al (2009) Value of perineural edema/inflammation detected by fat saturation sequences in lumbar magnetic resonance imaging of patients with unilateral sciatica. Acta Radiol 50(2):205-211

Publisher's Note Springer Nature remains neutral with regard to jurisdictional claims in published maps and institutional affiliations.

\section{Affiliations}

\section{Niek Djuric ${ }^{1}$ (1) - Xiaoyu Yang ${ }^{1} \cdot$ Raymond W. J. G. Ostelo $^{2,3}$ - Sjoerd G. van Duinen ${ }^{4} \cdot$ Geert J. Lycklama à Nijeholt ${ }^{5}$. Bas F. W. van der Kallen ${ }^{4}$. Wilco C. Peul ${ }^{1,5}$. Carmen L. A. Vleggeert-Lankamp ${ }^{1}$}

Niek Djuric

n.djuric@lumc.nl

1 Department of Neurosurgery, Leiden University Medical Center, Albinusdreef 2, 2300 RC Leiden, The Netherlands

2 Department of Epidemiology, VU Medical Centre, Amsterdam, The Netherlands
3 Department of Health Sciences, Faculty of Science, Amsterdam Movement Sciences Research Institute, Vrije Universiteit, Amsterdam, The Netherlands

4 Department of Pathology, Leiden University Medical Center, Leiden, The Netherlands

5 Haaglanden Medical Centre, The Hague, The Netherlands 